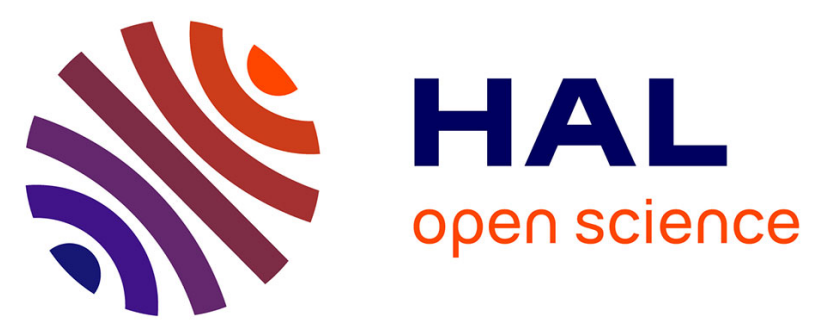

\title{
Sense, enrich and classify: The scanmaris workshop for assessment of vessel's abnormal behavior in the EEZ
}

Florent Jangal, Marie-Annick Giraud, Michel Morel, Jean-Pierre Mano, Aldo Napoli, Anne Littaye

\section{- To cite this version:}

Florent Jangal, Marie-Annick Giraud, Michel Morel, Jean-Pierre Mano, Aldo Napoli, et al.. Sense, enrich and classify: The scanmaris workshop for assessment of vessel's abnormal behavior in the EEZ. OCEANS 2008, Sep 2008, Quebec, Canada. 4 p. - Article number 5151852 - ISBN 978-142442620-1, 10.1109/OCEANS.2008.5151852 . hal-00569320

\section{HAL Id: hal-00569320}

https://hal-mines-paristech.archives-ouvertes.fr/hal-00569320

Submitted on 13 Nov 2012

HAL is a multi-disciplinary open access archive for the deposit and dissemination of scientific research documents, whether they are published or not. The documents may come from teaching and research institutions in France or abroad, or from public or private research centers.
L'archive ouverte pluridisciplinaire HAL, est destinée au dépôt et à la diffusion de documents scientifiques de niveau recherche, publiés ou non, émanant des établissements d'enseignement et de recherche français ou étrangers, des laboratoires publics ou privés. 


\title{
Sense, Enrich and Classify: the ScanMaris Workshop for Assessment of Vessel's Abnormal Behavior in the EEZ
}

\author{
F. Jangal ${ }^{\#}$ (ONERA), M.A. Giraud (SOFRESUD), M. Morel (DCNS), J.P. MANO (IRIT), \\ A. NAPOLI (Mines ParisTech), Anne LITTAYE (ECOMER) \\ http://www.irit.fr/scanmaris/ \\ ${ }^{\#}$ florent.jangal@onera.fr
}

\begin{abstract}
Constant monitoring of the Exclusive Economic Zone cannot be performed only using high performance sensors. On the one hand, all available information on the observed area as juridical history of vessels or delineation of fishing zone is not necessarily measurable. On the other hand, even if the large amount of available information could be caught out they would be useless if none thorough sorting and analysis are carried on. So, we propose to sense vessel trail in Exclusive Economic Zone, to enrich the trail with the relevant non-measurable information and to use this material to classify vessel behaviors. The main issues of this approach are the selection of the relevant details which point to uncommon behaviors and the definition of the criteria which allow differentiating uncommon behavior and fraudulent one. Thus, the challenge is to deal with multidisciplinary information (i.e. bathymetry, radar tracks, insurance database, weather, etc.) and multi-domain criteria (i.e. juridical, behavioral, zonal, temporal, etc.). The architecture presented here aims to overcome both the issues.
\end{abstract}

\section{INTRODUCTION}

Constant growth of world maritime transport and significant economic stakes of territorial water management have prompted the international community to invest in maritime global security research. In this context, the ScanMaris project, which is founded by the French National Research Agency (ANR), aims to continuously monitor activities in the Exclusive Economic Zone (EEZ) and detect abnormal behavior using both observation systems and external data sources.

The ScanMaris software workshop is based on the assumption that global surveillance of the EEZ cannot only use target detection systems. Indeed, whatever the performances of tracking algorithms are, they cannot estimate how suspicious the vessel tracks are. Some external information, like insurance database or transported goods, can improve behavior analysis.

We propose to collect numerous information on the observed area (i.e. vessel locations and tracks, weather conditions, previous committed offenses, destination, etc.) in

We would like to thank the French National Research Agency for its financial contribution to the project, regional safety and security centers (DAM and CROSS) for their support and all members of the project's steering committee for their support and advices. order to create an enriched map of the scene. Then, the map is analyzed and abnormal behaviors are picked out.

ScanMaris project can be though as a four layers system: observation of maritime activities with several kind of sensor, fusion of sensed data, enrichment of information with heterogeneous sources (e.g. EQUASIS safety-related base, weather condition, LLOYDS insurance base, history of vessels, delimitation of maritime zones, etc.) and classification of the overall behavior. However, as explained thereafter, the enriched map is the essential compound of the workshop and it is the central element of the project architecture.

The specification phases have been carried out until mid July 2008 and the project has now moved to its first development phase.

\section{SCANMARIS ARCHITECTURE}

\section{A. End User Needs}

Several discussions with the end users have permit to accurately defined ScanMaris outlines. These exchanges have also been useful to identify the succession of events which could describe abnormal behaviors.

Final project architecture and rules to be implemented in order to trig an alert have been derived from the analysis of end users needs.

\section{B. General Architecture}

The key element of the project is the enriched map (EM) which contains all information about vessels (i.e. position, insurance, class, route, etc.) and about scene layout (i.e. weather, registered maritime areas, bathymetry, etc.).

As shown in Fig. 1, all project elements are linked with EM. EM is a dynamic space-time object. Indeed, all events leaved on the map (e.g. new point of a vessel trail) have a position, a date, an identifier and a validity duration. The map must manage itself the evolution of events (e.g. decreasing of significance, storage, etc.). EM has also to select the relevant information to be sent to other compounds (e.g. vessels in vicinity, past anomaly, etc.) 


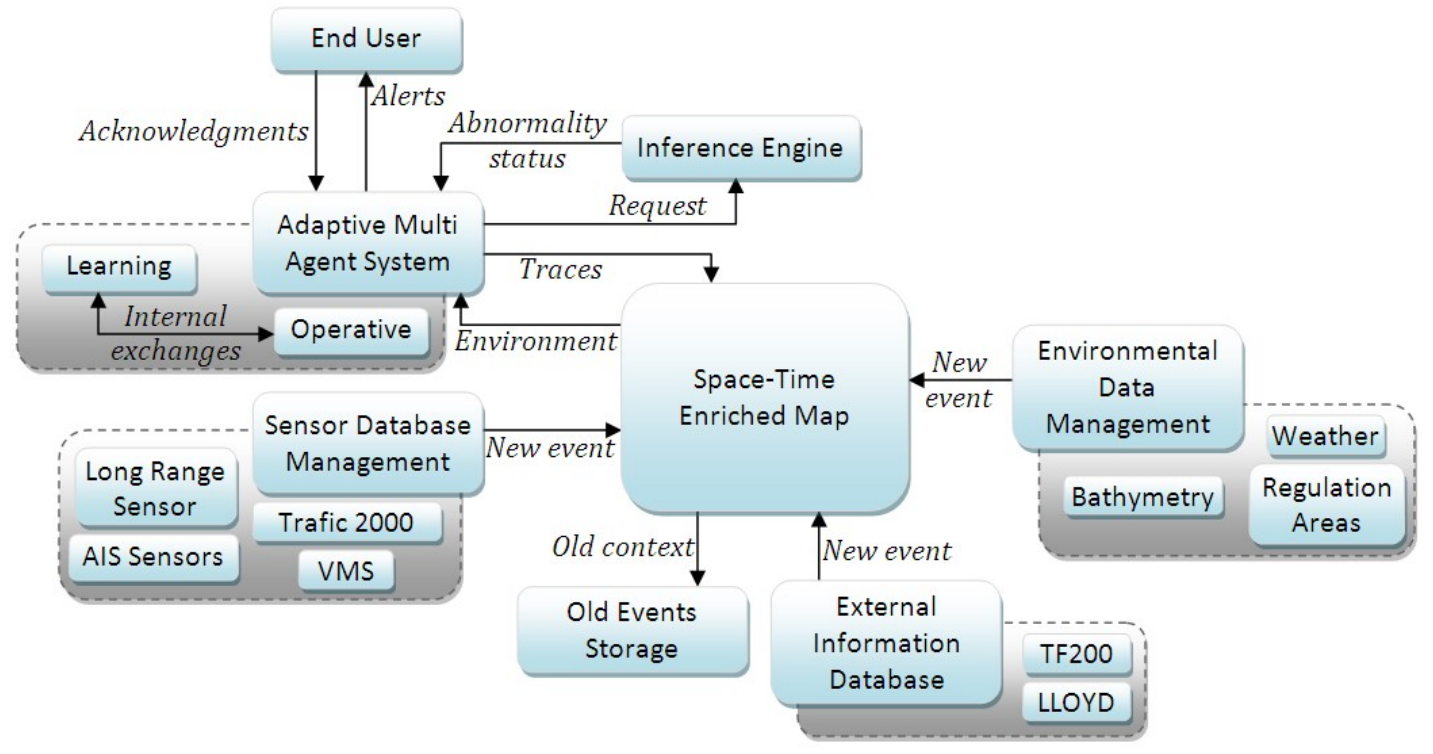

Figure 1. ScanMaris architechture.

1) Environmental Data Management: data dealing with bathymetry, weather, etc. are gathered by this module. If significant modifications of current data state occur, updated information is sent to the EM.

2) External Information Database: insurance or vessel history information is collected here. The main databases used by this module are the LLOYD insurance database and the TF2000 vessel database.

3) Old Event Storage: this is the "mass storage" of the project. All space-time events (e.g. vessel trail plus history of alert) can be stored in this module when they are no longer enough relevant to be kept in the EM.

4) End User: all the EM events are available to the end user. Tree level of alert can be sent to him: vigilance, pre-alert and alert. The user has the possibility to indicate to the system if an alert is unjustified or if the system has missed an abnormal behavior.

5) Inference Engine (IE): IE states either the normality or the abnormality of events. IE will not analyze the entire enriched map: this would be needlessly time consuming since fraud is a marginal activity. IE only answers to the requests send by Adaptive Multi-Agent Systems (AMAS) which extract relevant details.

6) Adaptive Multi Agent System: AMAS is the cognitive part of the project. Each vessel is associated to an agent. Each agent analyses its environment and significant modification of its environment highlights by the EM. Each agent leaves traces containing its current state and get traces of vessels in its vicinity area which contain current and former state of other agents. Dealing with this information, AMAS extract unusual event and request the analysis of inference engine.

7) Sensor database management: several sensors are used in the project. Some of them like the long range sensor, which is a High Frequency Surface Wave Radar (HFSWR), and the
Automatic Identification System (AIS) receivers are operated by consortium member. Only data of other sensors are available (e.g. Vessel Monitoring System or VMS database).

\section{HFSWR AND AIS}

High frequency surface wave radar (HFSWR) is a low cost system for the surveillance of the exclusive economic zone (EEZ). Since HFSWR operates in the HF band and uses surface waves, it is not limited by the radio horizon. Then, it is possible to cover an area of a few hundred kilometers over $120^{\circ}$ azimuth.

The Automatic Identification System (AIS) is principally used for identifying and locating vessels. Vessels can send and receive via AIS transponder their identification, position, course, and speed. AIS communications are located in the VHF-band. So, the coverage is limited by the radio horizon and antennas must be placed as high as possible. Nevertheless, it is estimated that more than 40,000 ships use AIS equipments.

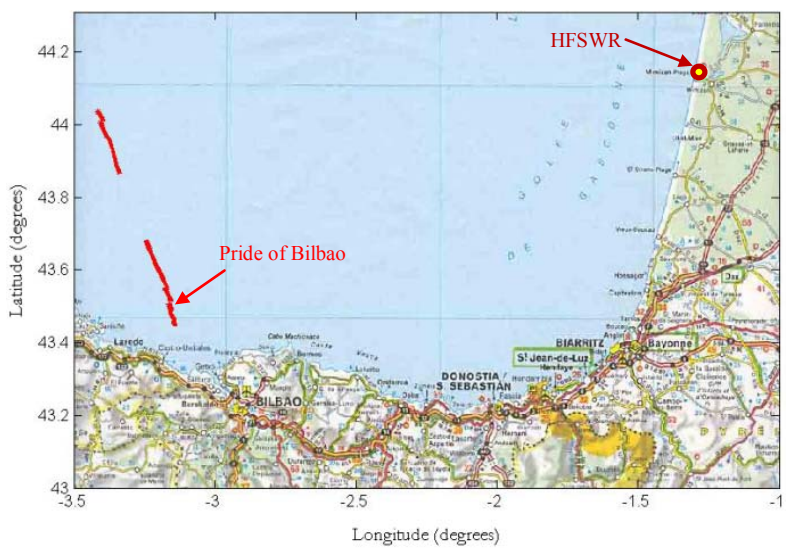

Figure 2. Tracking of the Pride of Bilbao ferry. 


\section{The HFSWR Operated by the ScanMaris Project}

1) The Radar: the HFSWR demonstrator is located inside a trial centre in the southwest of France (Centre d'Essais des Landes).

The radar transmitting system consists in a digital signal generator, a power amplifier (over $1.5 \mathrm{~kW}$ CWRMS), band pass filters and a biconical antenna.

The use of a digital generator allows synthesizing the signal directly in the HF band and modifying the transmitting wave form. In particular, it is possible to generate a multiple carrier frequencies signal.

The receiving system is made up of an array of 16 receiving antennas (more than $200 \mathrm{~m}$ long and over $10 \mathrm{~m}$ height) which is around $150 \mathrm{~m}$ away from the transmitting antenna, 16 simple receiving heads (no frequency transposition) and 16 digital receivers.

The digital receivers give the complex radar data (i.e. after a Hilbert transform) without any analog frequency transposition.

2) Main Feature: in order to maximize the excitation of surface waves the antennas have to be located very close to the shoreline. Moreover, we have installed the antennas at the bottom of a sand hill so that the back lobes should be minimal.

The spacing between the receiving antennas and their height are optimized for carrier frequencies from $5 \mathrm{MHz}$ to $10 \mathrm{MHz}$. Nevertheless, good results are obtained beyond $10 \mathrm{MHz}$.

The coverage range is over $200 \mathrm{~km}$ within an azimuth sector of about $\pm 45^{\circ}$ while the radar cell is $5 \mathrm{~km}$ deep (i.e. the radar bandwidth is $30 \mathrm{kHz}$ ) and around 10 degrees wide (Fig. 2).

3) AIS Preliminary Tests: some measurements with AIS has been done in the Biscay Bay (Fig.3). Depending on maritime traffic and willingness of crews to turn on AIS, variable number of vessels can be tracked.

4) Association of HFSWR and AIS Tracks: in order to avoid redundant information, AIS and HFSWR tracks must be associated. Thus, only one event will be sent to EM even if a vessel signal is probe by the two sensors.

The main rough tasks concerning this association are the correlation of asynchronous information about the same vessel and the factoring in false AIS position.

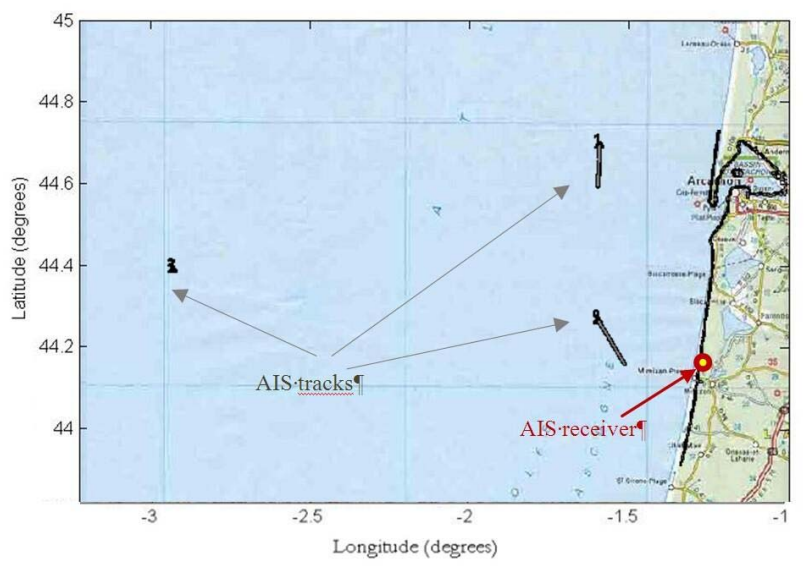

Figure 3. AIS tracks.

\section{AMAS}

Adaptive Multi-Agent Systems seems to be well-suited to extract uncommon behaviors. There are numerous similarities between ScanMaris issues and some AMAS issues. That is, extract some distinctive behaviors or features from a global scene (i.e. EM) where each element is autonomous (i.e. vessels).

Vessels, which can be thought as cooperative agents, affect the environment by leaving traces. Conversely, each agent is influenced by the environment, in particular, by the traces of other agents. Agents also get space-time information from the enriched map.

1) Inference Engine and AMAS: as mentioned before, the Adaptive Multi-Agent System can generate tree alert levels: vigilance, pre-alert, alert. Alerts are triggered according to abnormality status sent by IE as well as agent states. Nevertheless, an alert is not linked to one particular event but result from the accumulation of various anomalies in multiple agents.

Each agent, just like a vessel, explores a part of the enriched map. Agents evenly compare their nominal parameters (i.e. weather, position, etc.) with the information of the EM. If parameters and EM information diverge each agent request an abnormality status to the inference engine. As a result, IE send to each agent the pointed out unusual situations.

Using all agent information the AMAS analyze suspicious combination of anomalies. Moreover, traces leaved by agents contain unusual situations. Thus, suspicious event can also be identified by the agents themselves when they analyses all traces in their vicinity area.

2) Learning and Operative AMAS: obviously AMAS must have the opportunity to continuously learn which behavior should be pointed out or not. So, the workshop contains two AMAS: a learning and an operative AMAS

The operative AMAS uses the current know-how to evolve the agent states according to EM modifications. The learning AMAS is able to make its know-how progress. Besides, the learning AMAS is enable only when an acknowledgment is sent by the user. Then, the last events plus the user information are analyzed again in order to update the knowhow.

3) Traces: traces leaved by agents on the EM contain a date, an intensity or a persistence duration, a location and the identifier of the considered vessel.

The enriched map will use traces to send information about vessels in the vicinity of each agent traces.

\section{UPCOMING PROJECT PHASE}

The workshop elements as well as communication protocol or resources access strategy are now accurately defined. The development phase, which has started on July 2008, will first focus on the implementation of the proposed architecture, AMAS programming and real data acquisition.

Trials of each project compound will be done using the collected data. However, during a test cycle it is difficult to 
adjust algorithms only with a set of data where only few interesting case might be present. So, we are considering the opportunity to simulate complex scenarios in order to test each element, notably the AMAS.

At the end of the trial phase we except to be able to assess the relevance of specified rules, the efficiency of the chosen inference engine (i.e. data-driven engine with classical RETE algorithm), convergence of AMAS and contribution of HFSWR.

\section{CONCLUSION}

Global security of the Exclusive Economic Zone is a present challenge since over $90 \%$ of world trade travels by sea. We think that the transversal approach of ScanMaris will improve the EEZ management.

The key points of the projects are: the fusion of several sensor information, in particular data provided by High Frequency Surface Wave Radar; the continuous update of an enriched map using heterogeneous sources; the extraction of uncommon events performed by Adaptive Multi-Agent
Systems and Inference Engines which state the abnormality of each pointed out uncommon behaviors.

The project had begun since January 2008, the development phase has started since mid July 2008, the first global trial will be done in Marsh 2009 and final demonstration is scheduled on December 2009.

\section{REFERENCES}

[1] "Review of maritime transport", United Nations Conference on Trade and Development, Geneva, 2007, http://www.unctad.org/en/docs/ rmt2007 en.pdf

[2] M. Menelle, G. Auffray, F. Jangal, "Full Digital High Frequency Surface Wave Radar: French Trials in the Biscay Bay", Proceedings of IEEE Radar 2008, Adelaide, September 2008.

[3] G. Di Marzo Serugendo, M.-P. Gleizes and A. Karageorgos, "Self organisation and emergence in multi-agent systems: An overview", Informatica, Vol 30, No 1, pp 45-54, January 2006.

[4] J. Iris, A. Napoli and F. Guarnieri, "Evaluation of Spatial Multidimensional Approach for the Analysis of Disaster Data", Proceedings of the 25th Urban Database Management Symposium, Denmark, 2006. 